\title{
A Reduced Complexity of Vahedi's Tag Estimation Method for DFSA
}

\author{
Sanika K. Wijayasekara ${ }^{1, a}$, Annur Robithoh ${ }^{2, \mathrm{~b}}$, Pruk Sasithong ${ }^{3, \mathrm{c}}$, Pisit Vanichchanunt ${ }^{3, \mathrm{~d}, *}$, \\ Suvit Nakpeerayuth ${ }^{1, \mathrm{e}}$, and Lunchakorn Wuttisittikulkij ${ }^{1, \mathrm{f}, *}$ \\ 1 Department of Electrical Engineering, Faculty of Engineering, Chulalongkorn University, Bangkok 10330, \\ Thailand \\ 2 Faculty of Information and Communication Technology, Universiti Tunku Abdul Rahman, Kuala \\ Lumpur, Malaysia \\ 3 Department of Electrical and Computer Engineering, Faculty of Engineering, King Mongkut's University \\ of Technology, North Bangkok, Thailand \\ E-mail: awijayasekarasanika@gmail.com, brobithoh@utar.edu.my, cpruk.sasithong@gmail.com, \\ dv_pisit@hotmail.com (Corresponding author), ensuvit@chula.ac.th, \\ fwlunchak@chula.ac.th (Corresponding author)
}

\begin{abstract}
In order to calculate the number of tags in a radio frequency identification (RFID) system, several tag estimation methods have been investigated in literature and most of the available estimation methods need the overall knowledge of idle, success and collision slots of the previous frame to carry out the tag estimation process. In this article, we present three techniques to reduce the complexity of Vahedi's tag estimation for tag King Mongkut's University of Technology North Bangkokcollision resolution in RFID systems using dynamic frame slotted ALOHA. Our modified and useful approach considers the information about only the number of empty, successful or colliding slots in the previous frame for the tag estimation. Three decision rules were obtained by maximizing the likelihood of success, idle and collision which helps in the reduction of complexity substantially. However, the accuracy of estimation decreases for success-only and idle-only methods while the collision-only method gives a consistent and lower estimate error when the frame sizes and the number of tags increase.
\end{abstract}

Keywords: DFSA, tag estimation, collision resolution, RFID.

ENGINEERING JOURNAL Volume 21 Issue 6

Received 28 August 2017

Accepted 16 October 2017

Published 31 October 2017

Online at http://www.engj.org/

DOI:10.4186/ej.2017.21.6.111 


\section{Introduction}

\subsection{RFID Technology}

In automatic identification industry, ubiquitous tagging forms a unique identification for any object in Internet of Things (IoT). For the process of human identification, there exist bio-metric (finger print, DNA, Iris detection) as well as document based identification (national identity card, passport, etc.) to distinguish humans. Similarly, in object identification tags or codes are formed and appear on everything such as, in healthcare, aerospace, banking and etc to automatically identify the things [1,2]. Bar code, RFID (radio frequency identification), fingerprints, voice recognitions are some of those generally used for identification technologies. Among the above-mentioned item identification techniques, RFID technology has emerged as an attractive solution for object identification, and it gets more attention as an alternative to the conventional techniques such as bar codes, in many industries around the world [3].

RFID is a wireless technology to track objects using radio frequency signals. As depicted in Fig. 1, an RFID system is a combination of RFID technology and computer technology. It encloses with an application in a computer, a reader and several tags which relies on exchanging command between the reader and tags. The readers are capable of keeping track and monitoring the tagged objects through a shared wireless channel and the tags are attached to the objects which are needed to be identified [4]. RFID tags can be either active or passive, where the passive tags do not contain any internal power source and the active tags are either partially or fully battery powered.

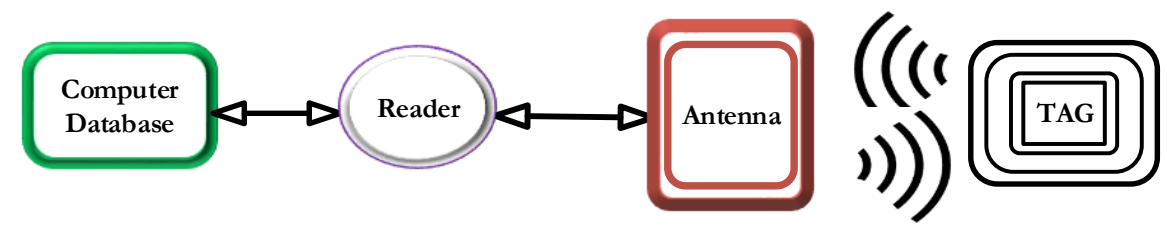

Fig. 1. A basic RFID system.

In RFID systems, the reader sends a query command to tags within its interrogation zone with a defined frame size and tags then reply by sending a Radio Frequency (RF) signal on its randomly selected slot. As a result, each slot in the frame can contain RF signals from one tag, more than one tag or no signal at all. This represents the success slot $S$, collision slot $C$ and idle slot $E$ respectively. When there is a collision, the reader will not be able to distinguish these signals and identify each individual tag correctly. To overcome this collision problem, several anti-collision algorithms have been proposed in literature [5-9], some of which are presented in the following section in greater details.

\subsection{Collision Resolution Algorithms}

In RFID systems, collision resolution protocols can be categorized into three types, namely tree, ALOHA and query tree (QT) based algorithms [10]. The tree algorithms subgroup the collided tags recursively through a tree structure until each leaf node of the tree contains one tag or none. In binary tree algorithm, for example, the collided tags are split into two subgroups. If one of these two subgroups or both subgroups still contain collision, the collided tags in each subgroup is further split into two subgroups. This procedure is carried out repeatedly until all collisions are completely resolved, and hence each tag is uniquely identified. M-ary tree concept is also available in literature where the collided tags are divided into M number of subgroups [10-13] instead of two as in the binary tree algorithm.

The ALOHA based protocols are categorized into pure ALOHA, slotted ALOHA and frame slotted ALOHA (FSA). In slotted ALOHA, the time is divided into slots and corresponding tags can send the information at the beginning of each slot. In contrast, for the frame slotted ALOHA, the time is structured as series of frames, each of which is composed of a fixed number of slots. Each tag can transmit the information only at one slot in each frame. Dynamic frame slotted ALOHA (DFSA) [14-20], follows the similar concept as FSA but here the size of the frame is dynamic, i.e., frame can have different number of slots. The frame sizes are determined dynamically with respect to the information about remaining unidentified tags, number of collided slots and so on. 
Query Tree protocols divide the tags into two groups after analyzing the tag IDs. Initially, the reader queries with a prefix (which is a basically a bit string) to collect the information of the tags in its interrogation area. The tags which are having the matching prefixes in their IDs reply to the reader. When the reader experiences more than one reply, reader queries for one more bit longer prefix. However, query tree mechanism is hard to implement in the EPC (Electronic Product Code) Global standard [21, 22].

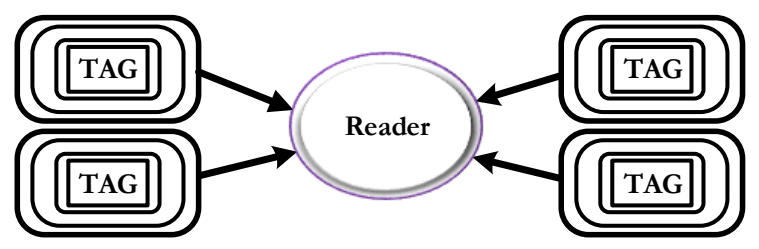

Fig. 2. Four tags communicating with a reader simultaneously: -Tag-Tag Collision.

In the beginning of a tag identification period, the reader does not have any information about the number of tags in its interrogation zone. To achieve fast tag identification, it is important for the reader to continuously learn about the actual number of tags in the system during the entire tag identification process, usually through some estimation methods. In [15] the author proposed a novel tag estimation method based on a posteriori probability applicable for DFSA protocols. However, a serious problem in the proposed model is that feedbacks from each slot are assumed independent. Subsequent research work found that such an assumption is not valid and the corrected model is given in [23] with a substantial increase of computation complexity.

In this article, we form three decision rules to reduce the complexity in tag estimation method introduced by the authors in [23] called as Vahedi's method. The main contribution of Vahedi's method is to correct a posterior analytical model proposed by Chen [15] and improve the performance of DFSA.

DFSA protocols need to adjust the next frame size by estimating the collided tags in the current frame. Figure 3 illustrates an example of DFSA with adaptive frame sizes of 4 and 3 and the principle procedures of DFSA algorithm in RFID can be stated as follows:

Step 1: The Reader broadcast a "Query $(Q)$ " command indicating the frame size as $2^{Q}(Q \in\{1,2, \ldots, 16\})$

Step 2: After receiving the Query command, each of the tag in reader's interrogation zone selects a slot between 1 to $2^{Q}$ range and transmits ID during the selected time slot.

Step 3: Based on the received information from the tags the reader can identify the idle slots where no ID is sent, collision slots where more than one ID are sent and success slots where only one ID is sent.

Step 4: Upon identifying the number of idle, success and collision slots the reader classifies the frame size for the next frame.

Step 5: These steps are followed until the number of collision slots becomes zero.

In literature, many tag estimation methods are available and majority of the estimation techniques consider the number of success $-S$, idle - $E$ and collision - $C$ slots in a previous frame, with the length of $L$ when estimating the number of tags. The Schoute's algorithm given in [14], the author used the information of $S$ and $C$ slots from the previous frame in his computation. Meanwhile, in Chen's [15], Vahedi's [23], and Vogt's [24] methods, the values of $S, E$ and $C$ are utilized to estimate the remaining tags in the system. However, to the best of our knowledge, RFID tag estimation using only on one type of feedback has never been investigated. Therefore, to investigate the impact of using a single feedback type, in this work, we propose three tag estimation algorithms which rely only on one type of feedback information which is $S, E$, or $C$. In Section 3 and 4, we show that the proposed methods with single feedback type can reduced the complexity of the recently introduced mathematical model in [22] related to tag estimation. Further, we compare the tag estimation performance of our newly proposed algorithms with four well known tag estimation methods which are described in Section 2. The rest of the paper is organized as follows. Section 2 describes the related existing tag estimation mechanisms. Section 3 provides the analytical models of the proposed methods and the results and discussions are given in Section 4 . We conclude our work in Section 5. 


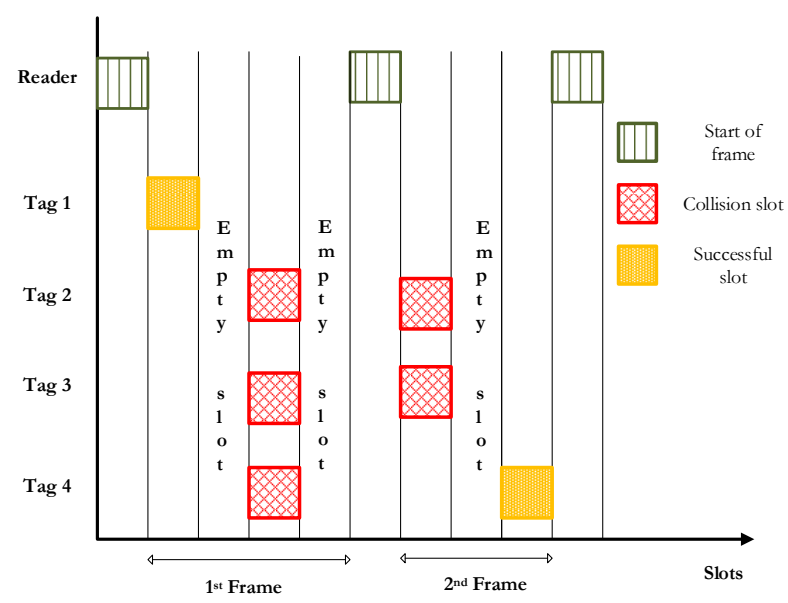

Fig. 3. Example of DFSA protocol with different frame length of 4 and 3, and 4 tags in the interrogation area.

\section{Tag Estimate Methods}

\subsection{Schoute's Algorithm}

The author in [14] introduced a very simple, fast and fairly accurate tag estimation method for DFSA algorithm based on $S$ and $C$ with the backlog estimator of $2.39 \times C$. The estimated number of tags $\bar{n}$ in the previous frame is given as,

$$
\bar{n}=S+2.39 C
$$

This method is accurate when the number of tags in each slot follows a Poisson distribution with an integer mean.

\subsection{Vogt's Algorithm}

In [24], the author obtained a tag estimation model based on binomial distribution in slot occupancy. For the given number of slots, $L$ in a frame and number of tags, $N$, the binomial probability that $i$ out of $N$ tags transmit their ID to a slot with access probability of $1 / L$ is given by,

$$
B_{N, 1 / L}(i)=\left(\begin{array}{c}
N \\
i
\end{array}\right)\left(\frac{1}{L}\right)^{i}\left(1-\frac{1}{L}\right)^{N-i}
$$

According to (2) the expected values of number of empty $a_{e}$, successful $a_{s}$, and collision $a_{c}$ slots are respectively derived as follows.

$$
\begin{gathered}
a_{e}=L B_{N, 1 / L}(0) \\
a_{s}=L B_{N, 1 / L}(1) \\
a_{c}=L\left(1-a_{e}-a_{s}\right)
\end{gathered}
$$

In this work, by assuming that at least $S+2 C$ tags are initially in the system, the minimum mean square error or minimum distance between the vector of the expected values and the vector of the read results as shown in Eq. (6) is used to estimate the number of tags in the system. 


$$
\bar{n}=\min _{N}\left|\left(\begin{array}{l}
a_{e} \\
a_{S} \\
a_{c}
\end{array}\right)-\left(\begin{array}{l}
E \\
S \\
C
\end{array}\right)\right|
$$

\subsection{Chen's Algorithm}

In [15], the author derived a posterior probabilistic model by considering binomial distribution of idle $\left(p_{e}\right)$, success $\left(p_{s}\right)$ and collision $\left(p_{c}\right)$ slots which occur in the previous frame as follows:

$$
\begin{gathered}
p_{e}=\left(1-\frac{1}{L}\right)^{N} \\
p_{s}=\left(\frac{N}{L}\right)\left(1-\frac{1}{L}\right)^{N-1} \\
p_{c}=1-\left(1-\frac{1}{L}\right)^{N}-\left(\frac{N}{L}\right)\left(1-\frac{1}{L}\right)^{N-1}
\end{gathered}
$$

The author assumed that the three outcomes of one read cycle: empty, successful and collision are independent and derived a posterior probability equation with multinomial distribution as shown in Eq. (10)

$$
P(E, S, C)=\frac{L !}{E ! S ! C !} p_{e}^{E} p_{s}^{S} p_{c}^{C}
$$

By maximizing a posterior probability expressed in Eq. (10), the estimated number of tags are approximated as follows:

$$
\bar{n}=\arg \max _{N} P(E, S, C \mid N)
$$

However, the three outcomes of success, collision and idle are not independent to each other. In Section 2.4 , the better a posterior probabilistic model is further discussed.

\subsection{Vahedi's Algorithm}

In [23], the authors reflect better approach of a posterior probability by assuming that tags in the frame are multinomial distributed and are mutually dependent for different slot types. As illustrated in Fig.4, a frame structure of $E$ empty slot in the first part of the structure by following $S$ success slots and $C$ collision slots in the last section of the structure is being considered to derive the correct a posterior probability close form formula shows in Eq. (12).

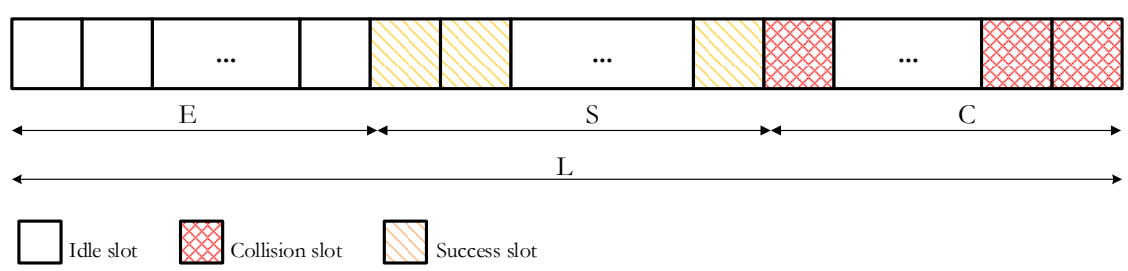

Fig. 4. The Frame structure considered in the analytical model of Vahedi [23].

$$
P(E, S, C)=\left(\frac{L !}{E ! S ! C !}\right) P_{1}(E) P_{2}(S \mid E) P_{3}(C \mid E, S)
$$


The probability of observing empty slots in the first part of the frame can be expressed as

$$
P_{1}(E)=\left(1-\frac{E}{L}\right)^{N}
$$

Next, the probability of observing successful slots in the second part of the frame when empty slots in the previous step is expressed as in (14), where the detail of its derivation is given in [23].

$$
\begin{aligned}
P_{2}(S \mid E) & =\left(\begin{array}{c}
N \\
S
\end{array}\right)\left(\frac{S}{L-E}\right)^{S}\left(1-\frac{S}{L-E}\right)^{(N-S)} \frac{S !}{S^{S}} \\
& =\left(\begin{array}{c}
N \\
S
\end{array}\right)\left(\frac{(L-E-S)^{(N-S)}}{(L-E)^{N}}\right) S !
\end{aligned}
$$

Finally, the probability of observing collision slots in the last part of the frame when empty slots and successful in the previous step is expressed as in (15), where the detail of its derivation is given in [23].

$$
\begin{aligned}
P_{3}(C \mid E, S)=\sum_{k=0}^{C} \sum_{\nu=0}^{C-k}(-1)^{(k+\nu)}\left(\begin{array}{l}
C \\
k
\end{array}\right)\left(\begin{array}{c}
C-k \\
v
\end{array}\right) \\
\times \frac{(N-S) !}{(N-S-k) !} \frac{(C-k-v)^{(N-S-k)}}{C^{(N-S)}}
\end{aligned}
$$

\section{Proposed Methods}

In this article, we propose three different decision rules to estimate the number of tags by considering the likelihood of only on one feedback type at a time. Three different closed form formulas are derived following the procedures given in [23] and we estimate the number of tags by maximizing the probability of empty, success, and collision.

\subsection{The Proposed Analytical Model Considering Only E}

In this approach, only the number of empty slots is used to estimate the number of tags in the system by maximizing the probability of empty slots in a frame. Here we use the frame structure with $E$ empty slot in first part of the frame structure and rest of $L-E$ slots varies between $S$ success slots and $C$ collision slots as illustrated in Fig. 5.

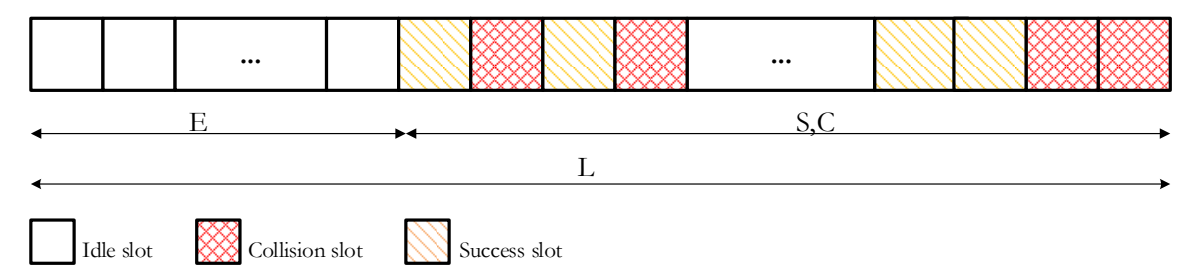

Fig. 5. The frame structure used to analyse the tag estimation based only on idle slots.

The derived closed-form formula for $P(E)$ is

$$
P(E)=\frac{L !}{E !(L-E) !} P_{1}(E) P_{2}(S, C \mid E)
$$

$P_{2}(S, C \mid E)$ in Eq. (17) represents the probability of $S$ successes and $C$ collisions incurring in the last part of the frame given that the first part of the frame is occupied by empty slots as follows: 


$$
P_{2}(S, C \mid E)=\frac{g_{N}(L-E, 1)}{(L-E)^{N}}
$$

where $g_{N}(L-E, 1)$ gives the number of acceptable success or collision events incurring in the last part of the frame structure. $(L-E)^{N}$ indicates different ways of distributing $N$ number of tags among $(L-E)$ slots. In order to derive an expression for $g_{N}(L-E, 1)$, we follow the classical urn model described in [25], where $\alpha, m$ and $s$ denote the number of balls, the number of urns and the minimum number of balls in each urn, respectively. As expressed in Eq. (18) and Eq. (19), [25] suggested two closed form recursive expressions for $g_{\alpha}(m, s)$ to calculate the values recursively.

$$
\begin{gathered}
g_{\alpha}(m, s)=m g_{\alpha-1}(m, s)+m\left(\begin{array}{c}
\alpha-1 \\
s-1
\end{array}\right) g_{\alpha-s}(m-1, s) \\
g_{\alpha}(m, s)=\sum_{k=0}^{m}(-1)^{k}\left(\begin{array}{l}
m \\
k
\end{array}\right) \frac{\alpha !}{(s-1) !^{k}(\alpha-s k+k) !} \\
\times g_{\alpha-s k+k}(m-k, s-1)
\end{gathered}
$$

The Eq. (18) is analysed based on three logical constraints given below:

a) if $(\alpha \neq 0)$ and $(m=0)$, then $g_{\alpha}(m, s)=0$;

b) if $(\alpha<m s)$, then $g_{\alpha}(m, s)=0$;

c) if $(m=1)$ and $(\alpha \neq 0)$ and $(\alpha \geq m s)=0$, then $g_{\alpha}(m, s)=1$;

By following Eq. (21) and Eq. (22), we can further simplify Eq. (18) and Eq. (19) and express a close form expression for $g_{\alpha}(m, s)$ with respective $\alpha, m$ and $s$ values, where $p_{0}(\alpha-k, m-k)$ shows the probability of having $(\alpha-k)$ tags and $(m-k)$ slots when all the slots carry at least one tag.

$$
\begin{gathered}
p_{0}(\alpha-k, m-k)=\sum_{v=0}^{m-k}(-1)^{v}\left(\begin{array}{c}
m-k \\
v
\end{array}\right)\left(1-\frac{v}{m-k}\right)^{(\alpha-k)} \\
g_{\alpha-k}(m-k, 1)=p_{0}(\alpha-k, m-k)(m-k)^{\alpha-k} \\
g_{N}(L-E, 1)=(L-E)^{N} \sum_{\nu=0}^{L-E}(-1)^{v}\left(\begin{array}{c}
L-E \\
v
\end{array}\right)\left(1-\frac{v}{L-E}\right)^{N}
\end{gathered}
$$

Therefore, using Eq.(18), Eq.(19), Eq. (20) and Eq. (21) we can derive a non-recursive form for $g_{N}(L-E, 1)$ to identify the exact number of acceptable events by simply replacing $\alpha$ with $N, m$ with $L-E$ and $s$ with 1 .

The Eq. (22) can be analysed using the following two constraints.

a) if $(N \geq 0)$ and $(L-E=0)$, then $g_{N}(L-E, 1)=0$;

b) if $\left(N<2(L-E)\right.$, then $g_{N}(L-E, 1)=0$;

By substituting Eq. (13) and Eq. (17) into Eq. (16),

$$
P(E)=\frac{L !}{E !(L-E) !} \frac{g_{N}(L-E, 1)}{L^{N}}
$$


Hence, the decision rule to estimate of the number of tags by using the information about following idle slots only is achieved by maximizing the Eq. (23) and is given by

$$
\bar{n}=\arg \max _{N} P(E \mid N)
$$

\subsection{The Proposed Analytical Model Considering Only $S$}

In this section, we discuss the likelihood of success slots $S$. Similar to earlier discussions to model this concept we consider a frame structure with $S$ success slots in first part of the structure and rest of $L-S$ slots varies between $E$ empty slots and $C$ collision slots as illustrated in Fig. 6.

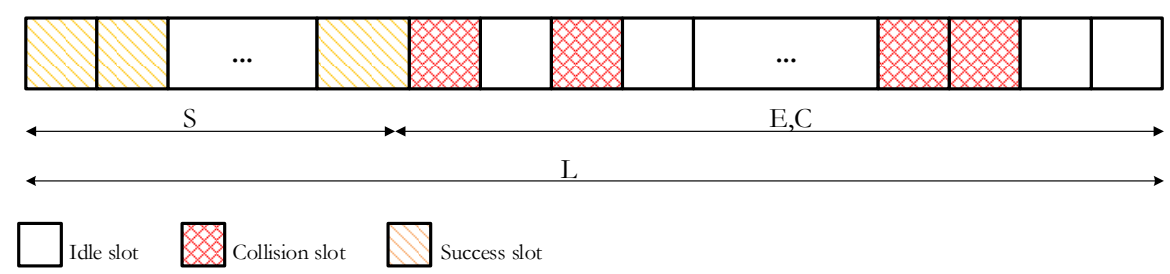

Fig. 6. The frame structure used to analyse the tag estimation based only on success slots.

By considering only the success, the likelihood probability success $P(S)$ can be modelled as,

$$
P(S)=\frac{L !}{S !(L-S) !} P_{1}(S) P_{2}(C, E \mid S),
$$

where,

$$
P_{1}(S)=\left(\begin{array}{c}
N \\
S
\end{array}\right)\left(\frac{S}{L}\right)^{S}\left(1-\frac{S}{L}\right)^{(N-S)} \frac{S !}{S^{S}}
$$

and

$$
P_{2}(C, E \mid S)=\sum_{E=0}^{L-S}\left(\begin{array}{c}
L-S \\
E
\end{array}\right)\left(1-\frac{E}{L-S}\right)^{N-S} \frac{g_{N-S}(L-S-E, 2)}{(L-S-E)^{N-S}} .
$$

In Eq. (26), the $\left(\frac{S}{L}\right)^{S}$ is the probability that the first $S$ slots out of $L$ slots are chosen by exactly $S$ tags. The $\left(1-\frac{S}{L}\right)^{(N-S)}$ is the probability that the remaining $L-S$ slots are selected by $N-S$ tags and $\frac{S !}{S^{S}}$ ensures that those $S$ tags selects the first $S$ slots in such a way that no empty or collision slots in-between. The $g_{N-S}(L-S-E, 2)$ gives the acceptable events that represent the ways to distribute $N-S$ tags among $L-S-E$ slots which carry at least 2 tags in a slot. Therefore, we can derive $g_{N-S}(L-S-E, 2)$ which is expressed in Eq. (28) using Eq. (18), Eq. (19), Eq. (20) by simply applying $s$ as 2 and $m$ as $L-S$-E. The $g_{N-S}(L-S-E, 2)$ can be analysed with following three defined constraints.

a) if $(N-S \geq 0)$ and $(L-S=0)$, then $g_{N} g_{N-S}(L-S, 2)=0$;

b) if $(N-S<2(L-S))$, then $g_{N-S}(L-S, 2)=0$;

c) if $(N-S \neq 0)$ and $L-S=1$ and $(N-S \geq 2(L-S))$, then $g_{N-S}(L-S, 2)=0$;

$$
\begin{aligned}
g_{N-S}(L-S-E, 2)=\sum_{k=0}^{L-S-E} \sum_{\nu=0}^{L-S-E-k}(-1)^{(k+\nu)}\left(\begin{array}{c}
L-S-E \\
k
\end{array}\right)\left(\begin{array}{c}
L-S-E-k \\
v
\end{array}\right) \\
\times \frac{N-S !}{(N-S-k) !}(L-S-E-k-v)^{(N-S-k)}
\end{aligned}
$$


Therefore, the decision rule to estimate the number of tags by using the information about the success slots only is derived by maximizing Eq. (25) and is given by

$$
\bar{n}=\arg \max _{N} P(S \mid N)
$$

\subsection{The Proposed Analytical Model Considering Only $C$}

To model the probability of collision slots the frame structure is given in Fig. 7 is considered, where the first part of the structure presents only the collision slots and rest of $L-C$ slots represents $S$ success slots and Eidle slots.

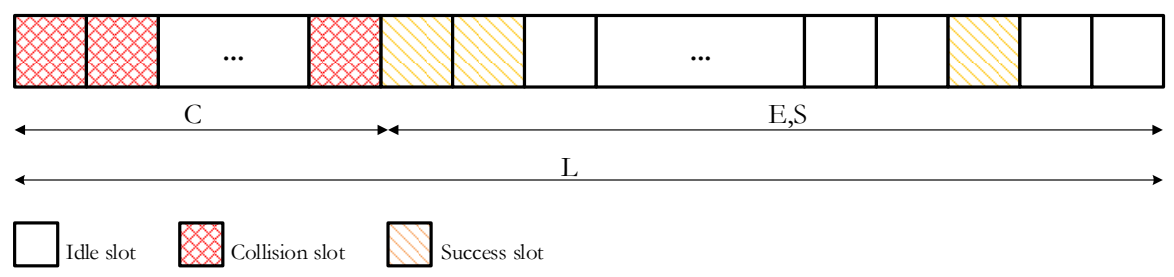

Fig. 7. The frame structure used to analyse the tag estimation based only on collision slots.

The $P(C)$ is given as

$$
P(C)=\frac{L !}{C !(L-C) !} \sum_{\substack{n=2 C \\ n>N-L+C}}^{n} P_{1}(C \mid n) P_{2}(S, E \mid n, C)
$$

where

$$
P_{1}(C \mid n)=\left(\begin{array}{c}
N \\
n
\end{array}\right) \frac{g_{n}(C, 2)(L-C)^{N-n}}{L^{n}}
$$

and

$$
P_{2}(S, E \mid n, C)=\frac{(L-C) !}{(L-C-N+n) !}\left(\frac{1}{L-C}\right)^{N-n} .
$$

In Eq. (31), $n$ indicates the number of collided tags where $\frac{g_{n}(C, 2)(L-C)^{N-n}}{L^{n}}$ is the probability of first part of the frame structure given in Fig. 7 contains only collided slots and $g_{n}(C, 2)$ follow the same process given in Eq. (19) with following constraints.
a) if $(n \neq 0)$ and $(C=0)$, then $g_{n}(C, 1)=0$;
b) if $(n<2 C))$, then $g_{n}(C, 1)=0$;
c) if $(n=0)$ and $(C=0)$, then $g_{n}(C, 1)=1$;

In Eq. (32), $(L-C)$ ! represents the number of ways of $S$ success slots and $E$ idle slots happen, while $(L-C-N+n)$ ! presents the number of ways of $C$ collision slots occur. $\left(\frac{1}{L-C}\right)^{N-n}$ shows the probability that the remaining $(N-n)$ tags are assigned to the remaining $L-C$ slots.

By substituting Eq. (31) and Eq. (32) in Eq. (30) the $P(C)$ can be derived as,

$$
P(C)=\frac{L !}{C !} \sum_{\substack{n=2 C \\
n>N-L+C}}^{N}\left(\begin{array}{c}
N \\
n
\end{array}\right) \frac{g_{n}(C, 2)}{L^{N}(L-C-N+n) !} .
$$


Therefore, the decision rule to estimate the number of tags by using the information about the following collision slots only is obtained by maximizing Eq. (33) and is given by

$$
\bar{n}=\arg \max _{N} P(C \mid N)
$$

\section{Results and Discussion}

Using Eq. (24), Eq. (29), and Eq. (34), we derived the lookup tables for the proposed idle-only, successonly and collision-only methods with respect to different frame sizes $(L=10,20,30,40,50)$ and number of estimated tags as shown in Table 1, Table 2 and Table 3 respectively. We can prove the simplicity of proposed approaches by comparing the proposed method's lookup tables with Chen [15] and Vahedi [23] method's lookup tables given in Table 4 and Table 5. As shown in Table 1 to 3 , the proposed approach has reduced the complexity of each lookup table by considering only one feedback type in tag estimation, where Chen [15] and Vahedi [23] required both success and collision.

Table 1. The estimated number of tags considering only $E$.

\section{\begin{tabular}{|l|c|c|c|c|c|c|c|c|c|c|c|}
\hline E & 0 & 1 & 2 & 3 & 4 & 5 & 6 & 7 & 8 & 9 & 10 \\
\hline$\hat{h}$ & 170 & 170 & 170 & 170 & 170 & 170 & 170 & 170 & 170 & 170 & 0 \\
\hline
\end{tabular}}

(a) $L=10$.

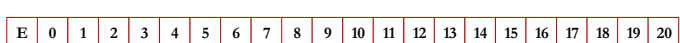
\begin{tabular}{|l|l|l|l|l|l|l|l|l|l|l|l|l|l|l|l|l|l|l|l|l|l|}
\hline$\hat{n}$ & 170 & 170 & 170 & 170 & 170 & 170 & 170 & 170 & 170 & 170 & 170 & 170 & 170 & 170 & 170 & 170 & 170 & 170 & 170 & 170 & 0 \\
\hline
\end{tabular}

(b) $L=20$.

\begin{tabular}{|l|l|l|l|l|l|l|l|l|l|l|l|l|l|l|l|l|l|l|l|l|l|l|l|l|l|l|l|l|l|l|l|}
\hline E & 0 & 1 & 2 & 3 & 4 & 5 & 6 & 7 & 8 & 9 & 10 & 11 & 12 & 13 & 14 & 15 & 16 & 17 & 18 & 19 & 20 & 21 & 22 & 23 & 24 & 25 & 26 & 27 & 28 & 29 & 30 \\
\hline & 7 & & 10 & 7 & 10 & 17 & & & & &
\end{tabular}

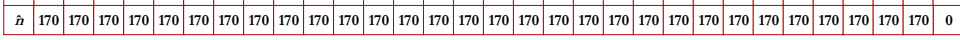

(c) $L=30$.

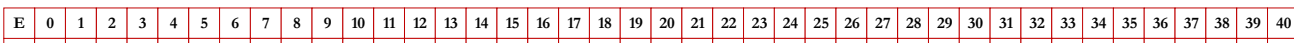

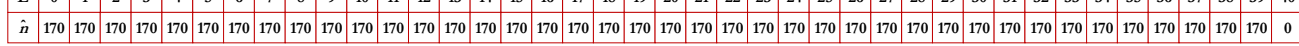

(d) $L=40$.

\begin{tabular}{|l|l|l|l|l|l|l|l|l|l|l|l|l|l|l|l|l|l|l|l|l|l|l|l|l|l|l|l|l|l|l|l|l|l|l|l|l|l|l|l|l|l|l|l|l|l|l|l|l|l|l|l|}
\hline $\mathrm{E}$ & 0 & 1 & 2 & 3 & 4 & 5 & 6 & 7 & 8 & 9 & 10 & 11 & 12 & 13 & 14 & 15 & 16 & 17 & 18 & 19 & 20 & 21 & 22 & 23 & 24 & 25 & 26 & 27 & 28 & 29 & 30 & 31 & 32 & 33 & 34 & 35 & 36 & 37 & 38 & 39 & 40 & 41 & 42 & 43 & 44 & 45 & 46 & 47 & 48 & 49 & 50 \\
\hline & 17 & & & & & &
\end{tabular}

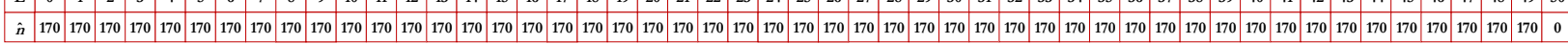

(e) $L=50$.

Table 2. The estimated number of tags considering only $S$.

\begin{tabular}{|l|l|l|l|l|l|l|l|l|l|l|l|}
\hline $\mathrm{S}$ & 0 & 1 & 2 & 3 & 4 & 5 & 6 & 7 & 8 & 9 & 10 \\
\hline & 17 & 17 & 17 & 170 & 170 & & & & \\
\hline
\end{tabular}

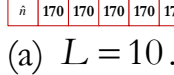

\begin{tabular}{|l|l|l|l|l|l|l|l|l|l|l|l|l|l|l|l|l|l|l|l|l|l|}
\hline $\mathrm{S}$ & 0 & 1 & 2 & 3 & 4 & 5 & 6 & 7 & 8 & 9 & 10 & 11 & 12 & 13 & 14 & 15 & 16 & 17 & 18 & 19 & 20 \\
\hline
\end{tabular}

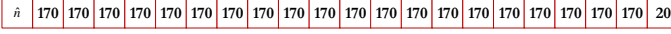

(b) $L=20$. 


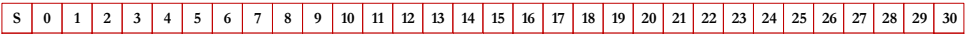

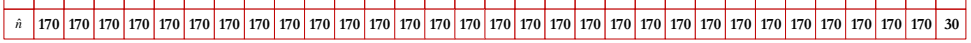

(c) $L=30$.

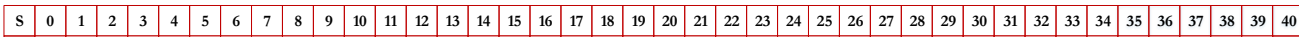

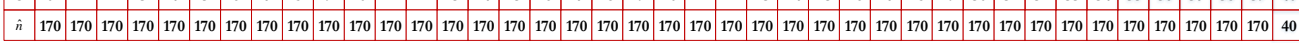

(d) $L=40$.

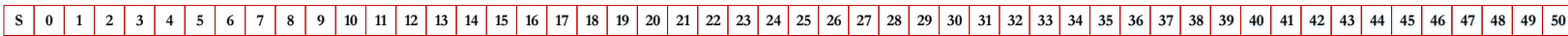

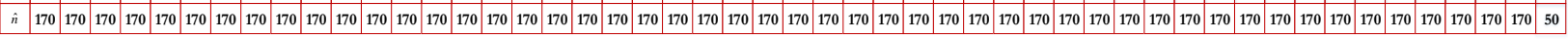

(e) $L=50$.

Table 3. The estimated number of tags considering only $C$.

\begin{tabular}{|l|l|l|l|l|l|l|l|l|l|l|l|}
\hline C & 0 & 1 & 2 & 3 & 4 & 5 & 6 & 7 & 8 & 9 & 10 \\
\hline & 1 & 5 & 8 & 11 & 13 & 6 & 1 & 24 & 2 & 38 & 170 \\
\hline
\end{tabular}

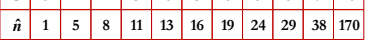

(a) $L=10$.

\begin{tabular}{|l|l|l|l|l|l|l|l|l|l|l|l|l|l|l|l|l|l|l|l|l|l|}
\hline $\mathrm{C}$ & 0 & 1 & 2 & 3 & 4 & 5 & 6 & 7 & 8 & 9 & 10 & 11 & 12 & 13 & 14 & 15 & 16 & 17 & 18 & 19 & 20 \\
\hline
\end{tabular}

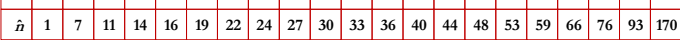

(b) $L=20$.

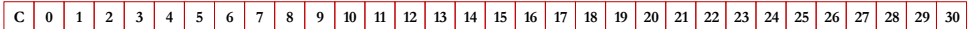

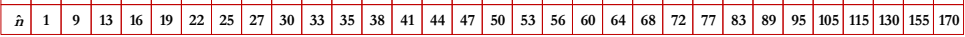

(c) $L=30$.

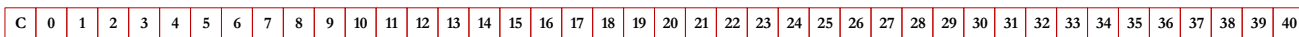

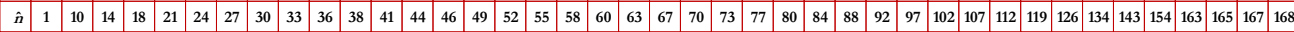

(d) $L=40$.

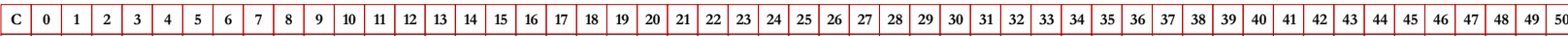

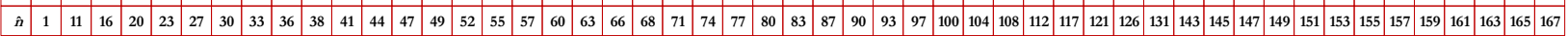

(e) $L=50$.

Table 4. The estimated number of tags following Chen's method [15] for $L=10$.

\begin{tabular}{|c|c|c|c|c|c|c|c|c|c|c|}
\hline C & 0 & 1 & 2 & 3 & 4 & 5 & 6 & 7 & 8 & 9 \\
\hline 1 & 3 & 3 & 4 & 5 & 6 & 7 & 8 & 9 & 10 & 11 \\
\hline 2 & 5 & 6 & 7 & 8 & 9 & 10 & 11 & 12 & 13 & - \\
\hline 3 & 7 & 8 & 9 & 10 & 11 & 12 & 13 & 14 & - & - \\
\hline 4 & 10 & 11 & 12 & 13 & 14 & 15 & 16 & - & - & - \\
\hline 5 & 12 & 14 & 15 & 16 & 17 & 19 & - & - & - & - \\
\hline 6 & 16 & 17 & 19 & 20 & 22 & - & - & - & - & - \\
\hline 7 & 20 & 22 & 23 & 25 & - & - & - & - & - & - \\
\hline 8 & 26 & 28 & 30 & - & - & - & - & - & - & - \\
\hline 9 & 35 & 38 & - & - & - & - & - & - & - & - \\
\hline 10 & 365 & - & - & - & - & - & - & - & - & - \\
\hline
\end{tabular}

Table 5. The estimated number of tags following Vahedi's method [23] for $L=10$. 


\begin{tabular}{|c|c|c|c|c|c|c|c|c|c|c|}
\hline C $^{S}$ & 0 & 1 & 2 & 3 & 4 & 5 & 6 & 7 & 8 & 9 \\
\hline 1 & 2 & 3 & 4 & 5 & 6 & 7 & 8 & 9 & 10 & 11 \\
\hline 2 & 4 & 5 & 6 & 7 & 8 & 9 & 10 & 11 & 12 & - \\
\hline 3 & 6 & 7 & 8 & 9 & 11 & 12 & 13 & 14 & - & - \\
\hline 4 & 9 & 10 & 11 & 12 & 14 & 15 & 16 & - & - & - \\
\hline 5 & 12 & 13 & 15 & 16 & 17 & 19 & - & - & - & - \\
\hline 6 & 15 & 17 & 18 & 20 & 21 & - & - & - & - & - \\
\hline 7 & 20 & 21 & 23 & 25 & - & - & - & - & - & - \\
\hline 8 & 26 & 28 & 30 & - & - & - & - & - & - & - \\
\hline 9 & 35 & 38 & - & - & - & - & - & - & - & - \\
\hline 10 & 170 & - & - & - & - & - & - & - & - & - \\
\hline
\end{tabular}

Our proposed collision-only, success-only and empty-only methods were simulated with the frame lengths of $L=10,20,30,40,50$ based on the Monte Carlo technique. We then compare the tag estimation error of our proposed algorithms with four well established algorithms namely Schoute, Vogt, Chen, and Vahedi algorithms. We define the estimate error as

$$
\text { tag estimate error }=\frac{|N-\bar{n}|}{N}
$$

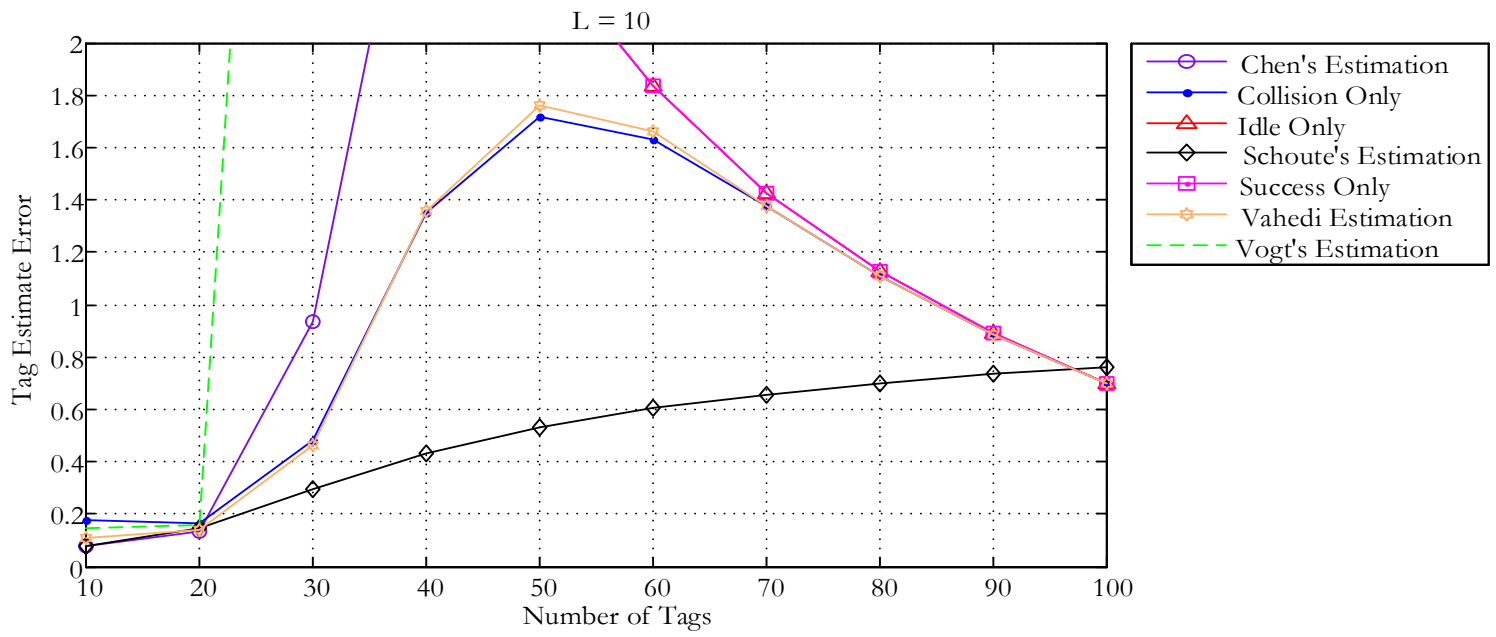

(a)

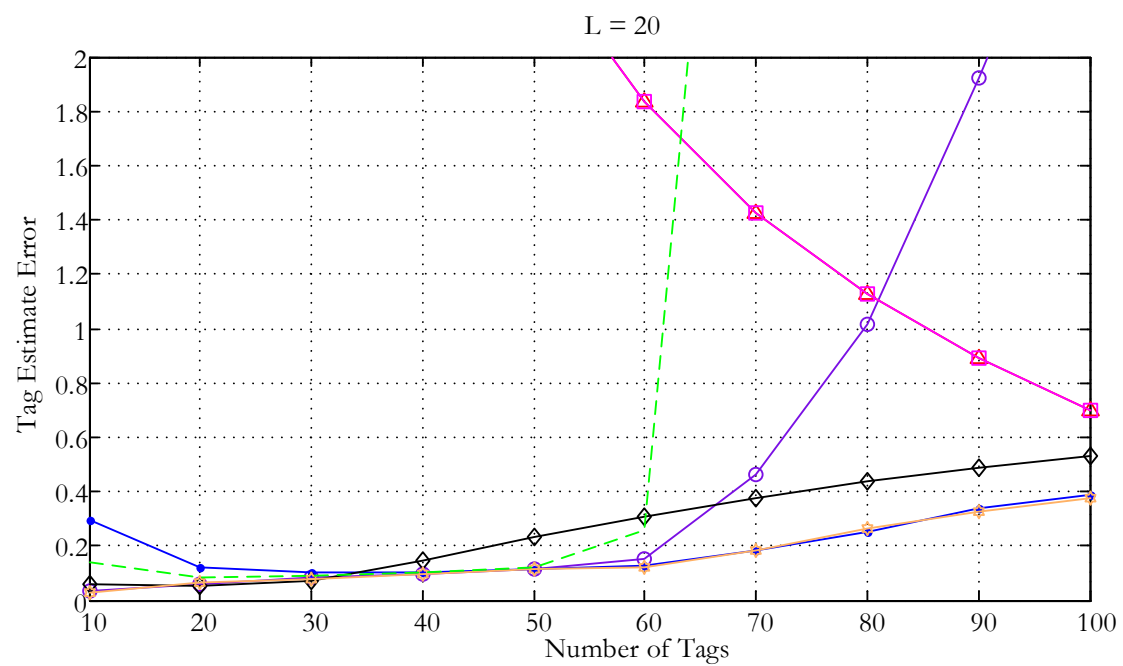

(b) 


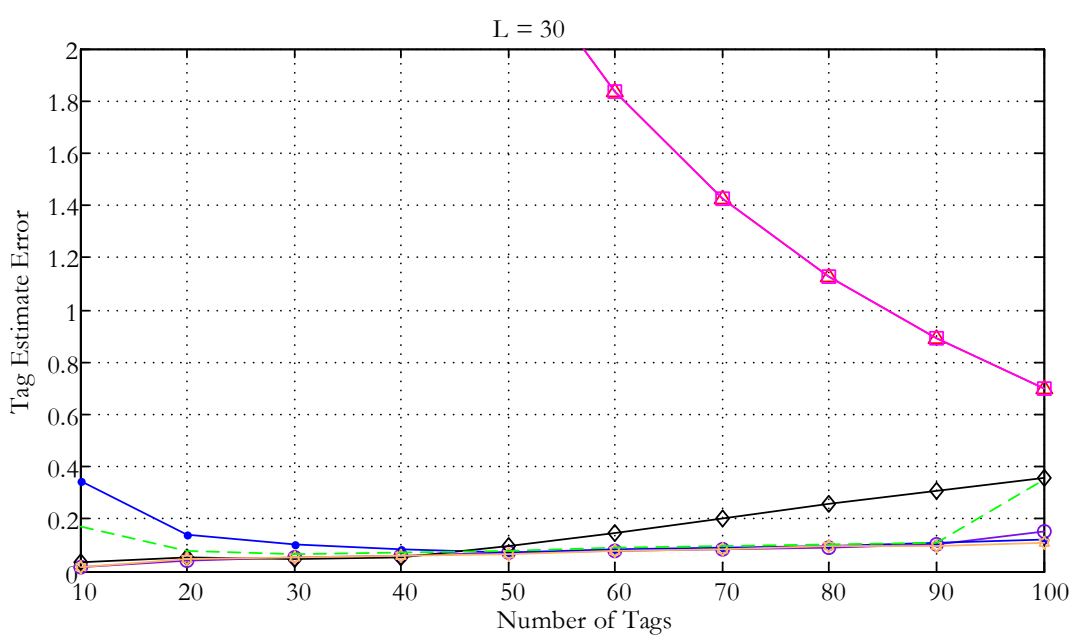

(c)

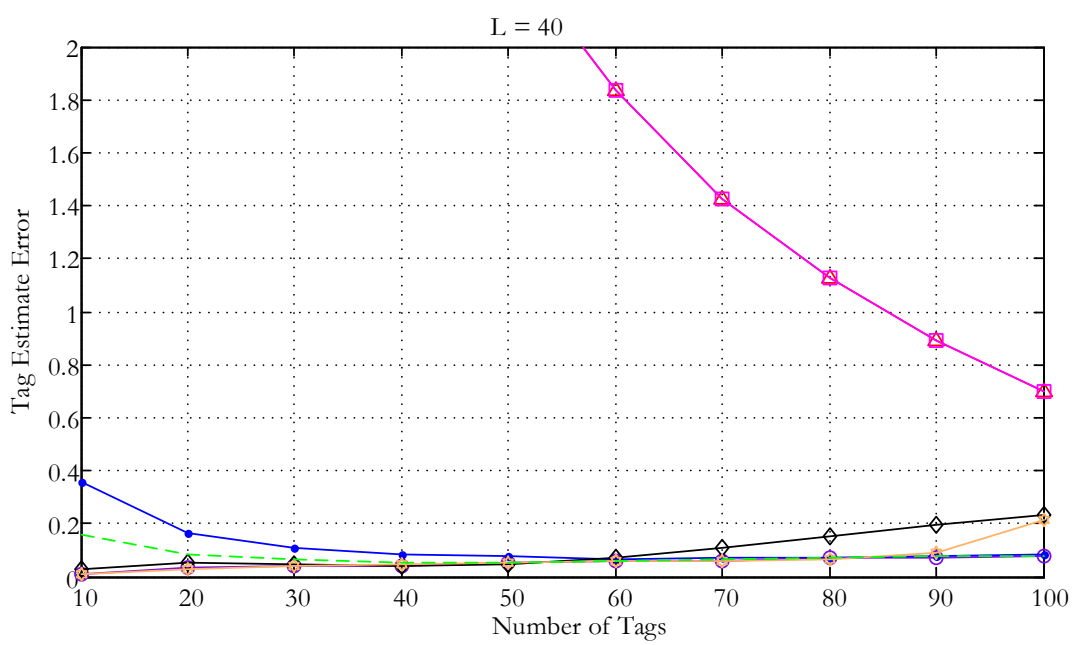

(d)

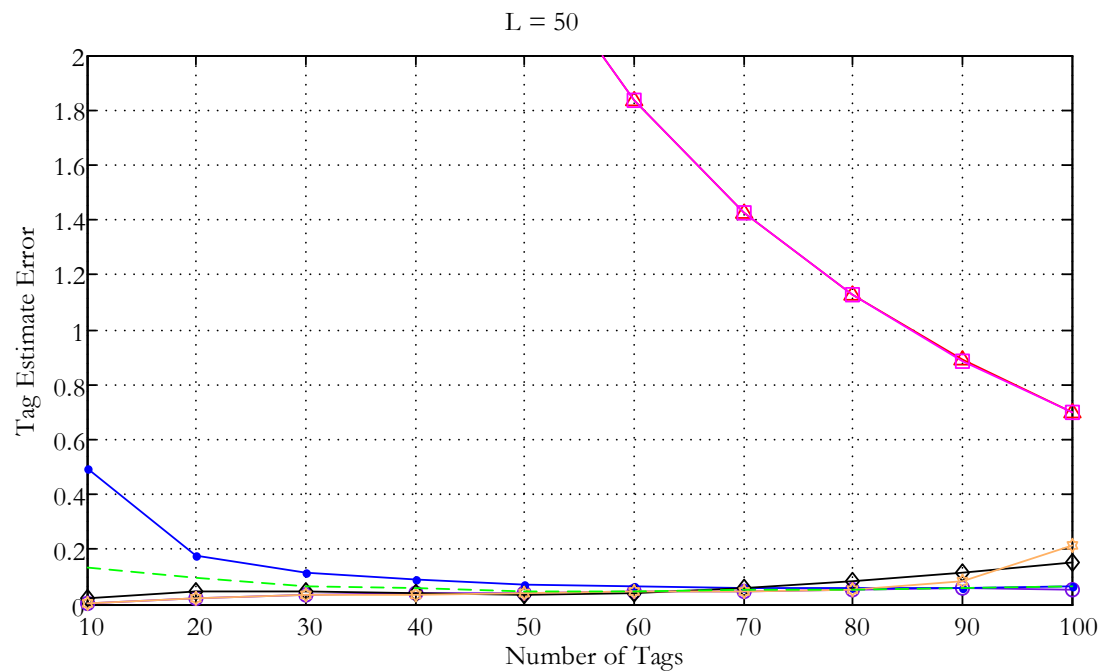

(e)

Fig. 8. Simulation results for tag estimate error with different frame sizes. 
For each technique, the estimation process was carried out for one frame and the resultant $S, C, E$ slot counts were used to evaluate each algorithm. The comparison results are shown in Fig. 8 with respect to different frame sizes.

As depicted in Fig. 8, the proposed collision-only method performs well in high collision situations. Note that when the number of tags is twice the frame size we can experience higher number of collisions than success and idle. Therefore, when number of tags is twice larger than the frame sizes the estimation error of collision only method in Fig. 8 is lower than the other two proposed methods. Apart from that, collision only method shows a similar curve as those of Vogt, Vahedi and Chen methods. The error rate is reduced and gives a consistent value for higher number of tags. However, by analyzing the aforementioned look up tables in Table 1, Table 2, Table 3, Table 4, and Table 5, we can assure that our proposed methods have reduced the complexity of Vahedi's approach by reducing number of tables.

\section{Conclusion}

We proposed a reduced-complexity tag estimation method based on Vahedi's a posterior analysis, which has three variants namely success-only, collision-only and idle-only. The mathematical analysis is carried out for each variant thereby achieving three different decision rules for tag estimation. Performance of four well known estimation algorithms in literature including Vogt's, Schoute's, Chen's and Vahedi's algorithms are considered and compared with our proposed techniques in terms of the error in tag estimation. Numerical results show that feedback information of success-only and idle-only is found to be insufficient to accomplish accurate estimation across different combination of the number of tags and slots. On the contrary collision-only estimation method performs well for both small and large number of tags, given different number of slots. When compared to other well-known methods the proposed collision-only method offers comparable performance. As our proposed method requires less feedback in tag estimation, the lookup table can be reduced to one dimensional as opposed to all other techniques that their lookup tables are two dimensional.

\section{Acknowledgments}

The authors would like to acknowledge the Graduate School of Chulalongkorn University, Thailand for the 100th anniversary Chulalongkorn University Fund for Doctoral Scholarship and $90^{\text {th }}$ Anniversary Chulalongkorn University Fund (Ratchdaphiseksomphot Endowment Fund). The first author would like to acknowledge the Department of Electrical Engineering Faculty of Engineering, Chulalongkorn University, Thailand for the Electrical Engineering Chulalongkorn University $\mathrm{PhD}$ (EECU-PhD) Honors Program Scholarship. The first author would like to express her sincere appreciation to Dr. Muhammad Saadi, Assistant Professor, Department of Electrical Engineering, University of Central Punjab for his kind support in revising this journal. Further, we are grateful to the referees for their insightful comments and suggestions to improve the quality of this paper.

\section{References}

[1] D. H. Shih, P. L. Sun, D. C. Yen, and S. M. Huang, "Taxonomy and survey of RFID anti-collision protocols," Computer Communications, vol. 29, no. 11, pp. 2150-2166, 2006.

[2] X. Su, C. C. Chu, B. S. Prabhu, and R. Gadh, "On the creation of automatic identification and data capture infrastructure via RFID," UCLA, 2007.

[3] C. Abraham, V. Ahuja, A. K. Ghosh, and P. Pakanati, "Inventory management using passive RFID tags: A survey," Department of Computer Science, The University of Texas at Dallas, 2002.

[4] R. Want, "An introduction to RFID technology," IEEE Pervasive Computing, vol. 5, pp. 25-33. 2006.

[5] S. Nakpeerayuth, L. Wuttisittikulkij, P. Vanichchanunt, W. Srichavengsup, N. Wattanamongkhol, R. Annur, M. Saadi, K. Wannakong, and S. Siwamogsatham, "Efficient medium access control protocols for broadband wireless communications," in Advanced Trends in Wireless Communications. InTech, 2011.

[6] Z. Tang and Y. He, "Research of multi-access and anti-collision protocols in RFID systems," in International Workshop on Anti-Counterfeiting, Security and Identification (ASID), Xiamen, Fujian, China, 2007, pp. 377-380. 
[7] P. Šolić, J. Radić, and N. Rožić, "Algorithm for deriving optimal frame size in passive rfid uhf class1gen2 standard using combinatorial model boundaries," Automatika, vol. 51, no. 255-263, pp. 255-263, 2010.

[8] J. L. Massey, "Collision-resolution algorithms and random-access communication," in Multi-User Communication Systems, vol. 265. Vienna: Springer, 1981, pp. 73-137.

[9] R. Raphael and S. Moshe, Multiple Access Protocols: Performance and Analysis, 1st ed. New York: SpringerVerlag, 1990.

[10] J. Su, Z. Sheng, D. Hong, and G. Wen, "An effective frame breaking policy for dynamic framed slotted aloha in RFID," IEEE Communications Letters, vol. 20, no. 4, pp. 692-695, 2016.

[11] P. Mathys and P. Flajolet, "Q-ary collision resolution algorithms in random-access systems with free or blocked channel access," IEEE Transactions on Information Theory, vol. 31, no. 2, pp. 217-243, Mar. 1985.

[12] J. Capetanakis, "Tree algorithms for packet broadcast channels," IEEE Transactions on Information Theory, vol. 25, pp. 505-515, 1979.

[13] H. Wu, Y. Zeng, J. Feng, and Y. Gu, "Binary tree slotted ALOHA for passive RFID tag anticollision," IEEE Transactions on Parallel and Distributed Systems, vol. 24, pp. 19-31, Jan. 2013.

[14] F. Schoute, "Dynamic frame length ALOHA," IEEE Transactions on Communications, vol. 31, no. 4, pp. 565-568, Apr. 1983.

[15] W. T. Chen, "An accurate tag estimate method for improving the performance of an RFID anticollision algorithm based on dynamic frame length ALOHA," IEEE Transactions on Automation Science and Engineering, vol. 6, no. 1, pp. 9-15, Jan. 2009.

[16] W. T. Chen, "A feasible and easy-to-implement anticollision algorithm for the EPCglobal UHF Class1 Generation-2 RFID Protocol," IEEE Transactions on Automation Science and Engineering, vol. 11, no. 2, pp. 485-491, Apr. 2014.

[17] P. Šolić, J. Radić, and N. Rožić, "Energy efficient tag estimation method for ALOHA-based RFID systems," IEEE Sensors Journal, vol. 14, no. 10, pp. 3637-3647, Oct. 2014.

[18] X. Jia, Q. Feng, and C. Ma, "An efficient anti-collision protocol for RFID tag identification," IEEE Communications Letters, vol. 14, no. 11, pp. 1014-1016, Nov. 2010.

[19] X. Jia, Q. Feng, and L. Yu, "Stability analysis of an efficient anti-collision protocol for RFID tag identification," IEEE Transactions on Communications, vol. 60, no. 8, pp. 2285-2294, Aug. 2012.

[20] X. Jia and Q. Feng, "An improved anti-collision protocol for radio frequency identification tag," International Journal of Communication Systems, vol. 28, no. 3, pp. 401-413, Feb. 2015.

[21] H. Gou, H. C. Jeong, and Y. Yoo, "A bit collision detection based query tree protocol for anticollision in RFID system," in IEEE 6th International Conference on Wireless and Mobile Computing, Networking and Communications, Niagara Falls, 2010.

[22] X. Jia and Y. Gu, "Standards and protocols for RFID tag identification," in Electronics, Communications and Networks IV. Taylor and Francis Group, 2015, pp. 581-855.

[23] E. Vahedi, W. S. Vincent, I. F. Blake, and R. K. Ward, "Probabilistic analysis and correction of Chen's tag estimate method," IEEE Transactions on Automation Science and Engineering, vol. 8, no. 3, pp. 659-663, Jul. 2011.

[24] H. Vogt, "Efficient object identification with passive RFID tags," in Proceeding Pervasive’02 Proceedings of the First International Conference on Pervasive Computing, August, 2002, pp. 98-113.

[25] J. Riordan, An Introduction to Combinatorial Analysis. New York: Wiley, 1985. 